\title{
Elderly quality of life impacted by traditional chinese medicine techniques
}

This article was published in the following Dove Press journal:

Clinical Interventions in Aging

30 September 2010

Number of times this article has been viewed

\author{
Helena A Figueira ${ }^{1,3,5}$ \\ Olivia A Figueira ${ }^{2}$ \\ Alan A Figueira ${ }^{1,4}$ \\ Joana A Figueira' \\ Tania S Giani ${ }^{1,3,5}$ \\ Estélio HM Dantas 3,5
}

'ABACO/Sohaku-in Institute, Rio de Janeiro, Brazil; 2IPEMED - Medical Research and Teaching Institute, Belo Horizonte, Brazil; ${ }^{2}$ LABIMH/ UNIRio - Laboratory of Biomedical Human Kinetics/Rio de Janeiro Federal University, Rio de Janeiro, Brazil; ${ }^{4} U F F$, Federal Fluminense University, Rio de Janeiro, Brazil; ${ }^{5}$ REMH - Euroamerican Network of Human Kinetics, Rio de Janeiro, Brazil

Correspondence: Helena A Figueira Estrada do Rio Morto, 197 bloco 10 casa 107, Recreio dos Bandeirantes, Rio de Janeiro,

ZIP 22783.210, Brazil

$\mathrm{Tel}+552178922264$

Fax +552124283528

Email helenafigueira@gmail.com
Background: The shift in age structure is having a profound impact, suggesting that the aged should be consulted as reporters on the quality of their own lives.

Objectives: The aim of this research was to establish the possible impact of traditional Chinese medicine (TCM) techniques on the quality of life (QOL) of the elderly.

Sample: Two non-selected, volunteer groups of Rio de Janeiro municipality inhabitants: a control group (36 individuals), not using TCM, and an experimental group (28 individuals), using TCM at ABACO/Sohaku-in Institute, Brazil.

Methods: A questionnaire on elderly QOL devised by the World Health Organization, the WHOQOL-Old, was adopted and descriptive statistical techniques were used: mean and standard deviation. The Shapiro-Wilk test checked the normality of the distribution. Furthermore, based on its normality distribution for the intergroup comparison, the Student $t$ test was applied to facets 2, 4, 5, 6, and total score, and the Mann-Whitney $U$ rank test to facets 1 and 3, both tests aiming to analyze the $P$ value between experimental and control groups. The significance level utilized was $95 \%(P<0.05)$.

Results: The experimental group reported the highest QOL for every facet and the total score.

Conclusions: The results suggest that TCM raises the level of QOL.

Keywords: quality of life, traditional chinese medicine, east-west medicine, WHOQOL-Old, elderly

\section{Introduction}

Traditional Chinese medicine (TCM), which is among the most ancient living traditions, ${ }^{1}$ is used almost worldwide nowadays, as there has been a global increase in demand for its treatment. In countries where the dominant health care system is based on allopathic medicine, or where TCM has not been incorporated into the national health care system, TCM is often termed "complementary", "alternative" or "non-conventional" medicine. ${ }^{2}$ Populational aging is bringing a greater incidence of chronic diseases, and TCM techniques offer the potential to deal with them. ${ }^{2}$ Traditional Chinese medicine, which is more than 2,500 years old, ${ }^{3}$ is practiced and taught in many health centers worldwide. In Brazil, some schools are traditional in this area, such as ABACO/Sohaku-in, which offers many techniques, including acupuncture, oriental massage, acupressure, herbal medicine, oriental dietetics, electro-acupuncture, and cranial acupuncture.

Quality of life (QOL) is based on both objective circumstances and subjective evaluations. Its subjectivity can be supported by objective factors, such as accomplishing 
one's socio-cultural goals of status, wealth, and physical wellbeing. ${ }^{4}$ It is conceptualized as a generic, multidimensional parameter, describing an individual's subjective perception of his/her physical and psychological health, as well as his/ her social functioning and environmental and general life status. $^{3}$ The complex and multifaceted concept of quality of life can be regarded as the outcome of advantages and disadvantages experienced over the course of life, depending on the satisfaction of social, economic, physical, emotional, psychic, and mental conditions, as stated by the World Health Organization (WHO). Its measurements have typically included a series of life domains: physical, emotional, social, environmental, and material..$^{5}$ Those who evaluate their QOL synthesize their experiences and perceptions. Those who achieve a successful synthesis experience a good QOL. ${ }^{6}$ This multidimensional concept, ${ }^{7}$ structured on a social and biological basis, and mediated by emotional, psychic, and mental conditions, is recognized as multidisciplinary, ${ }^{8}$ requiring multiple approaches from different theoretical angles.

The WHOQOL-Old questionnaire is a specific, complementary set of items recently developed by WHO to increase the specificity of quality of life measurement in older adults. It comprises six facets (sensory abilities; autonomy; past, present, and future activities; social participation; death and dying; intimacy), plus an overall score. ${ }^{9}$

QOL, self-evaluated via WHOQOL-Old by the elderly volunteer subjects randomly selected from inhabitants of Rio de Janeiro municipality who were treated either with TCM techniques or by non-TCM medicine, was investigated in an effort to establish the possible impact of TCM techniques on the QOL of the elderly.

\section{Preliminaries - subjects and methods Design of the research}

This study design is an enquiry descriptive model as ex post facto research.

\section{Universe}

The population of Rio de Janeiro City is composed of $6,093,472$ individuals, of which 233,085 are elderly; that is, $3.8 \%$ of the total population. ${ }^{10}$

\section{Data collection}

Prior to this study, two steps were taken: (i) approval was gained from the authority of the ABACO/Sohaku-in
Institute in order to apply the questionnaire to the experimental group; and (ii) terms of agreement were signed by the participants of both groups. The WHOQOLOld questionnaire was then applied. The inclusion criterion was that the participant must be a voluntary, elderly person living in Rio de Janeiro municipality. For the experimental group, there was also an inclusion criterion that the participant must have been treated with TCM techniques at the Sohaku-in Institute, and there was no exclusion criterion. For the control group, the exclusion criterion was that the participant must not be of a socio-economic level, or have any mental disorientation, that could distinguish them from the experimental group.

This essay complies with the ethical principles for research carried out on human beings laid down in the Helsinki Declaration. ${ }^{11,12}$ There is no external funding source for this research. The project has been approved by the Euroamerican Network of Kinesiology Research Ethics Committee.

\section{Sample}

The sample was composed of randomly-selected elderly (defined as aged over 60 years) volunteers living in Rio de Janeiro municipality. ${ }^{13}$ There were two groups: an experimental group treated with traditional Chinese medicine techniques at ABACO/Sohaku-in Institute as weekly outpatients for over three years; and a control group with no experience of TCM techniques. The experimental group comprised 28 non-selected elderly of both genders (mean age $=$ $73.0 \pm 8.3$ ). The control group comprised 36 non-selected elderly of both genders (mean age $=70.8 \pm 8.0$ ). The data were collected in October 2009 by the main author of this essay. Every individual who volunteered to answer the questionnaire in that month was accepted as part of our sample.

The protocol utilized for QOL observation was the WHOQOL-Old questionnaire, developed by WHO using a transcultural approach. ${ }^{14}$ It contains six facets of 4 items each: facet 1 on sensorial functioning; facet 2 on autonomy; facet 3 on future, present and past activities; facet 4 on social participation; facet 5 on death and dying; and facet 6 on intimacy. ${ }^{15}$ From the results, a total score (TS) is calculated in absolute and relative values. The validated Brazilian version of the WHOQOL-Old was used to avoid cultural variation. ${ }^{16}$ The questionnaire was individually administered, and the elderly individuals were asked to have in mind their own values, hopes, pleasures, and concerns, based on the previous two weeks. ${ }^{17}$ 
Table I Experimental group facets and total score descriptive statistics

\begin{tabular}{llllllll}
\hline & Facet I & Facet 2 & Facet 3 & Facet 4 & Facet 5 & Facet 6 & TS \\
\hline Mean & 17.78 & 17.22 & 16.06 & 16.00 & 15.44 & 15.67 & 16.36 \\
SD & 2.39 & 1.70 & 2.55 & 2.68 & 3.50 & 2.35 & 1.69 \\
\% Mean & 86.13 & 82.63 & 75.38 & 75.00 & 71.50 & 73.00 & 77.25 \\
S-W & 0.002 & 0.123 & 0.301 & 0.160 & 0.090 & 0.469 & 0.312 \\
\hline
\end{tabular}

Abbreviations: SD, standard deviation; S-W, Shapiro-Wilk normality test $P$ value; TS, total score.

\section{Data analysis}

Descriptive statistical techniques were used: mean standard deviations. The Shapiro-Wilk test was used to check the normality of the distribution (ie, Gaussian or not). In consequence, for the intergroup comparison, the Student $t$ test (for independent samples) and the Mann-Whitney U test were used to analyze the $P$ value for the facets plus total scores. The significance level utilized was $95 \%(P<0.05)$. The statistical results were presented in tables and graphs created by the Microsoft Excel ${ }^{\circledR}$ and IBM SPSS 17.0 programs.

\section{Results}

\section{Experimental group}

The empirical evaluation of elderly QOL, as facets and total scores, is presented in Table 1 . The TS was supported by facets 2, 3, 4 and 6. Score 5 (death and dying) reduced, and score 1 (sensorial functioning) raised the TS (Table 1).

\section{Control group}

Facets 1 (sensorial functioning) and 3 (future present and past activities) supported the total score, while facets 2 (autonomy) and 4 (social participation) reduced the mean, and facets 5 (death and dying) and 6 (intimacy) raised it (Table 2).

\section{Experimental versus control group}

The Shapiro-Wilk test was used to check the normality of the distribution. In consequence, for the intergroup comparison, the Student $t$ test for independent samples (a parametric test) was applied to facets 2, 4, 5, 6, and TS, while the Mann-Whitney U test (the strongest non-parametric test for independent samples) was applied to facets 1 and 3 (which did not present normality at $P<0.05$ ), both tests aiming to analyze the $P$ values for the facets and total scores between the experimental and control groups.

For facets 1,2 , and 4 , and the TS, there was a statistically significant difference. The scores for the experimental group showed a significant tendency towards a superior QOL. For the other facets $(3,5$, and 6$)$, there was no significant difference (Table 3).

The QOL total score and the QOL facet values of the experimental group were higher than those of the control group. Facet 1 (sensorial functioning) and facet 6 (intimacy) were respectively the highest QOL values in both the experimental and the control groups.

The overall result of this research was that the experimental group reported the highest QOL in every individual facet, and in the total score.

\section{Discussion}

TCM is a great tradition with a sound philosophical, experiential, and experimental basis. Increased side-effects of conventional medicine, the lack of curative treatment for several chronic diseases, the high cost of new drugs, microbial resistance, and emerging diseases are among the reasons for the renewed public interest in TCM. ${ }^{2}$ Many patients seek TCM techniques specifically for acupuncture treatment, which is reported to be helpful in some cases. ${ }^{18}$ Studies are expanding to assess the effectiveness of TCM as a treatment option. ${ }^{19,20}$ The unique characteristics of the clinical practice of TCM, such as pattern differentiation and individualization of treatment, make it difficult to translate its models. ${ }^{21}$

Table 2 Control group facets and total score descriptive statistics

\begin{tabular}{llllllll}
\hline & Facet I & Facet 2 & Facet 3 & Facet 4 & Facet 5 & Facet 6 & TS \\
\hline Mean & 13.62 & 13.10 & 14.24 & 13.48 & 13.52 & 15.29 & 13.87 \\
SD & 0.61 & 0.57 & 0.62 & 0.63 & 0.92 & 0.64 & 0.34 \\
\% Mean & 60.13 & 56.88 & 64.00 & 59.25 & 59.50 & 70.56 & 61.69 \\
S-W & 0.483 & 0.528 & 0.038 & 0.936 & 0.126 & 0.124 & 0.526 \\
\hline
\end{tabular}

Abbreviations: SD, standard deviation; S-W, Shapiro-Wilk normality test $P$ value; TS, total score. 
Table 3 Experimental versus control group quality of life central tendency measures

\begin{tabular}{llllllll}
\hline & Facet I & Facet 2 & Facet 3 & Facet 4 & Facet 5 & Facet 6 & TS \\
\hline$P$ value & $\mathbf{0 . 0 0 I}$ & $\mathbf{0 . 0 0 1}$ & 0.100 & $\mathbf{0 . 0 0 8}$ & 0.134 & $0.66 \mathrm{I}$ & $\mathbf{0 . 0 0 1}$ \\
\hline
\end{tabular}

Notes: Bold type indicates a significant statistical difference to $P<0.05$. The median was the chosen central tendency measure.

Abbreviation: TS, total score.

Facets 1 on sensorial functioning, 2 on autonomy, 4 on social participation, and TS, showing a significant tendency to be superior in the experimental group, confirm that TCM can have a positive effect on the autonomy of the elderly. On the other hand, adopting TCM techniques does not affect Facets 3 (future, present and past activities), 5 (death and dying), and 6 (intimacy), as was seen from the statistical results, which showed no significant difference for those facets between the control and the experimental groups.

Future, present and past activities, as well as death and dying, are philosophical themes that are considered neither in TCM treatments nor in Western medicine. Although a finding in India ${ }^{22}$ reports high QOL in both spheres compared to the other facets, this is considered to be a consequence of the subjects' philosophical beliefs.

With respect to intimacy, the literature shows greater satisfaction related to QOL than the other themes, ${ }^{23}$ which confirms this research finding. One of the transformations of late modernity is that people are architects of their own lives: there is an increased awareness among the general public of the importance in life of personal relationships. ${ }^{5}$ Older people must be recognized as human beings as a whole, accepting interpersonal relationships as a vital area in their lives too. ${ }^{24}$ Some scholars consider that becoming old is often equated with becoming lonely. There is no evidence for this belief, as loneliness might only be common among the very old. ${ }^{5}$

The control group, which had no TCM treatment experience, reported a QOL of $61.69 \%$, while the experimental group, treated with TCM, reported a $77.25 \%$ post-treatment QOL score, which is notably higher. The results of this pilot analysis suggest that more definitive research in this area is merited. ${ }^{25-28}$ Clinical studies that compare the effects of different treatment protocols are probably the most reliable source of evidence, and may also demonstrate a dose-response relationship, ${ }^{29}$ hence they are more than welcome.

The shift in age structure will have a profound impact on a broad range of economic, political, and social conditions. ${ }^{30}$ Policy-makers around the world are currently dealing with the effects of population aging. ${ }^{31}$ The views of the elderly must be sought in reaching operational definitions of quality of life, and as reporters on the quality of their own lives. The so-called "age burden" is a spurious concern that should not deter direct interviews with the elderly. ${ }^{32}$ There is a need to remind the general public that older people can still adapt well, enjoy life, and prosper in spite of the effects of aging. ${ }^{5}$

\section{Conclusion}

These preliminary data suggest a possible positive effect in QOL scores for those using TCM, as compared to the general population. It suggests that TCM might raise the level of QOL of the elderly. The overall conclusion of this research, reporting a higher QOL for those elderly who are treated with TCM, is favorable for the adoption of TCM techniques in the West, at least within the elderly population.

\section{Acknowledgments}

Estélio H M Dantas received a research grant from the National Research Council (CNPq).

\section{Disclosure}

The authors report no conflicts of interest in this work.

\section{References}

1. Patwardhan B, Warude D, Pushpangadan P, Bhatt N. Ayurveda and traditional Chinese medicine: a comparative overview. Evid Based Complement Alternat Med. 2005;2(4):465-473.

2. World Health Organization Programme on Traditional Medicine. WHO Traditional Medicine Strategy 2002-2005. Geneva: The World Health Organization; 2002.

3. Kim Y, Jun H, Chae Y, Park H, Kim B, Chang I, et al. The practice of Korean medicine: an overview of clinical trials in acupuncture. Evid Based Complement Alternat Med. 2005;2(3):325-352.

4. Zhang J, Huang H, Ye M, Zeng H. Factors influencing the subjective well being (SWB) in a sample of older adults in an economically depressed area of China. Arch Gerontol Geriatr. 2008;46(3):335-347.

5. Dykstra P. Older adult loneliness: myths and realities. Eur J Ageing. 2009;6(2):91-100.

6. Cummins R. The Validity and utility of subjective quality of life: a reply to Hatton and Ager. J Appl Res Intellect Disabil. 2002;15(3):261.

7. Kane RL, Kane RA, Bershadsky B, Degenholtz H, Kling K, Totten A, et al. Proxy sources for Information on nursing home residents' quality of life. J Gerontol B Psychol Sci Soc Sci. 2005;60(6):S318-S325.

8. Camfield L, Skevington SM. On subjective well-being and quality of life. J Health Psychol. 2008;13(6):764-775.

9. Chachamovich E, Fleck M, Laidlaw K, Power M. Impact of major depression and subsyndromal symptoms on quality of life and attitudes toward aging in an international sample of older adults. Gerontologist. 2008;48(5):593-602.

10. Instituto Brasilieiro de Geografia e Estatística. Demográfico I. Rio de Janeiro: Instituto Brasilieiro de Geografia e Estatística; 2000. 
11. World Medical Association. Declaration of Helsinki: ethical principles for medical research involving human subjects. Revised 2008. Seoul.

12. Goodyear M, Krleza-Jeric K, Lemmens T. The Declaration of Helsinki. BMJ. 2007;335(7621):624.

13. United Nations Department of Economic and Social Affairs: Population Division. Living Arrangements of Older Persons Around the World. New York: United Nations; 2005.

14. Hawthorne G, Davidson N, Quinn K, McCrate F, Winkler I, Lucas R, et al. Issues in conducting cross-cultural research: implementation of an agreed international protocol designed by the WHOQOL Group for the conduct of focus groups eliciting the quality of life of older adults. Qual Life Res. 2006;15(7):1257-1270.

15. Halvorsrud L, Kalfoss M, Diseth A, Halvorsrud L, Kalfoss M, Diseth A. Reliability and validity of the Norwegian WHOQOL-OLD module. Scand J Caring Sci. 2008;22(2):292-305.

16. Fleck MP, Chachamovich E, Trentini C. Development and validation of the Portuguese version of the WHOQOL-OLD module. Rev Saúde Pública. 2006;40:785-791.

17. Power M, Quinn K, Schmidt S. Development of the WHOQOL-old module. Qual Life Res. 2005;14(10):2197-2214.

18. Duncan B, White A, Rahman A. Acupuncture in the treatment of fibromyalgia in tertiary care - a case series. Acupunct Med. 2007;25(4): 137-147.

19. Lee M, Shin B, Ernst E. Acupuncture for treating menopausal hot flushes: a systematic review. Climacteric. 2009;12(1):16-25.

20. Wheeler J, Coppock B, Chen C. Does the burning of moxa (Artemisia vulgaris) in traditional Chinese medicine constitute a health hazard? Acupunct Med. 2009;27(1):16-20.

21. Zaslawski C. Ethical considerations for acupuncture and Chinese herbal medicine clinical trials: a cross-cultural perspective. Evid Based Complement Alternat Med. 2008; epub eCAM, doi:10.1093/ecam/ nen055.
22. Verma M. Working and non-working rural and urban older people: subjective well-being and quality of life. Indian J Gerontol. 2008;22(1): $107-118$.

23. Figueira H, Giani T, Beresford H, Ferreira M, Mello D, Figueira A, et al. Quality of life (QOL) axiological profile of the elderly population served by the Family Health Program (FHP) in Brazil. Arch Gerontol Geriatr. 2009;49(3):368-372.

24. Sumasy I. A biopsychosocial-spiritual model for the care of patients at the end of Life. Gerontologist. 2002;42:24-33.

25. Reynolds JA, Bland JM, MacPherson H. Acupuncture for irritable bowel syndrome - an exploratory randomised controlled trial. Acupunct Med. 2008;26(1):8-16.

26. Singh B, Wu W, Hwang S, Khorsan R, Der-Martirosian C, Vinjamury S, et al. Effectiveness of acupuncture in the treatment of fibromyalgia. Altern Ther Health Med. 2006;12(2):34-41.

27. Li P, Yang LP, Gong YW. Application of systems biology technology in research of traditional Chinese medicine. J Tradit Chin Med. 2009; 29(2):153-157.

28. Chen MR, Wang P, Cheng G, Guo X, Wei GR, Cheng XH. The warming acupuncture for treatment of sciatica in 30 cases. J Tradit Chin Med. 2009;29(1):50-53.

29. White A, Cummings M, Barlas P, Cardini F, Filshie J, Foster NE, et al. Defining an adequate dose of acupuncture using a neurophysiological approach - a narrative review of the literature. Acupunct Med. 2008; 26(2):111-120.

30. Tareque V. Bangladesh population ageing and life expectancies: 1950-2050. Indian J Gerontol. 2008;22(1):119-126.

31. Gierveld JdJ, Hagestad G. Perspectives on the integration of older men and women. Res Aging. 2006;28(6):627.

32. Kane RA. Definition, measurement, and correlates of quality of life in nursing homes: toward a reasonable practice, research and policy agenda. Gerontologist. 2003;43:28-36
Clinical Interventions in Aging

\section{Publish your work in this journal}

Clinical Interventions in Aging is an international, peer-reviewed journal focusing on evidence-based reports on the value or lack thereof of treatments intended to prevent or delay the onset of maladaptive correlates of aging in human beings. This journal is indexed on PubMed Central, MedLine, the American Chemical Society's 'Chemical

\section{Dovepress}

Abstracts Service' (CAS), Scopus and the Elsevier Bibliographic databases. The manuscript management system is completely online and includes a very quick and fair peer-review system, which is all easy to use. Visit http://www.dovepress.com/testimonials.php to read real quotes from published authors. 